\title{
Espacios públicos como áreas sensibles en el Centro Histórico de Quito
}

\section{Public spaces as sensitive areas in the Historic Center of Quito}

\author{
${ }^{1}$ Marianela Cruz Cabrera, arquitecta, PhD \\ Profesora, Arquitecta del Observatorio Urbano y del Paisaje \\ Facultad de Arquitectura y Urbanismo \\ Universidad Tecnológica Equinoccial, Quito-Ecuador. \\ 〈marianela.cruz@ute.edu.ec〉, ‘arqmarianelacruz@yahoo.es〉.
}

Recepción / Received: 30, 09, 2017

Aceptación / Accepted: 30, 11, 2017

Publicado / Published: 26, 12, 2017

Resumen: La ciudad tradicional y dentro de ella los centros históricos se constituyen en áreas muy sensibles de los contextos urbanos. Lugares que han sufrido transformaciones y en la actualidad son protagonistas de los efectos de la degradación de sus edificaciones, la segregación, la excesiva sobreexplotación, además de la agresión del tráfico automotor para lo cual no fueron diseñadas. El diseño de sus espacios públicos de conjunto con el paisaje urbano histórico actúan sin lugar a dudas como criterios fundamentales para estructurar y ordenar el uso y funcionamiento de estos lugares. El presente trabajo centra su atención en el área más central y asociada al período fundacional de la ciudad de Quito, específicamente, el Centro Histórico $(\mathrm{CH})$. El objetivo a alcanzar será mostrar problemáticas existentes a nivel del CHQ en general y en el área de estudio en específico, donde se continúa dando prioridad al tráfico automotor, la inseguridad se incrementa y sus dinámicas de uso continúan regidas por la economía terciaria y la informalidad, existe desarticulación de espacios públicos de alto valor simbólico, patrimonial e identitarios, así como la amenaza de que esta importante zona se convierta en un lugar meramente turístico y escenográfico. Se plantean posibles acciones que contribuyan a consolidar una red de espacios interconectados de lugares donde predominen los intereses del peatón Los centros históricos, su paisaje y la riqueza patrimonial que lo caracteriza se constituyen hoy en día en un valioso recurso, un derecho ciudadano en el cual hemos de apoyarnos para alcanzar la meta de la ciudad sostenible.

Palabras clave: centro histórico, espacios públicos, paisaje urbano histórico.

Abstract: The traditional city and its historical center are very sensitive areas of urban context. These places have passed through a lot of transformations and now they are the protagonists of buildings degradation, segregation, excessive overexploitation and the effects caused by the aggression of traffic, leaving aside for what they really were designed. One of the most important attribute of these spaces is the tangible reality of their historical urban landscape, now at days and in the future, it can be considered as a fundamental criterion to structure and order its use and operation. The purpose of this work is to show how the Historical Center of Quito does not attend correctly its landscape values, it continues giving priority to the automotor traffic, the insecurity increases and its dynamics of use continues to be governed by the tertiary economy, informality. 
Hence, the objective is to demonstrate the importance of preserving the existing values and the unavoidable need to achieve the rescue of their public spaces through the implementation of sensitive actions among which, the creation of an interconnected network stands out due to its connotation, an articulated of places where the interests of the pedestrian predominate in the historical center, their landscape and the patrimonial wealth that characterizes it are today a valuable resource, a citizen right in which we have to support us to reach the goal of the sustainable city.

Keywords: historical center, public space, Historic Urban Landscape.

\section{INTRODUCCIÓN}

La ciudad tradicional y dentro de ella los centros históricos se constituyen en áreas muy sensibles de los contextos urbanos, "el centro histórico trasciende el tiempo y el espacio", (Carrión 2009). En ellos se refleja la historia vivida del territorio y las personas que lo han habitado o visitado, una conjugación de pasado, presente y futuro en la que indiscutiblemente su influencia no puede ser negada. Son lugares que han sufrido transformaciones y, en la actualidad, son protagonistas de los efectos de la degradación de sus edificaciones, la segregación, la excesiva sobreexplotación de sus espacios y la agresión del tráfico automotor para lo cual no fueron diseñadas.

El paisaje urbano histórico y el diseño de los espacios públicos actúan sin lugar a dudas como criterios fundamentales para estructurar y ordenar el uso y funcionamiento de estas áreas centrales de la ciudad, ya que permite identificar aspectos significativos que son necesario mantener y conservar, no solo por su significado desde el punto de vista ambiental sino por los valores patrimoniales o culturales, que le son propios.

Los centros históricos, su paisaje y la riqueza patrimonial que lo caracterizan se constituyen hoy en día en un valioso recurso, un derecho ciudadano en el cual hemos de apoyarnos para alcanzar la meta de la ciudad sostenible. Atendiendo a estos planteamientos surge la interrogante sobre el futuro de estas áreas, cómo se desarrollarán y se desenvolverán sus dinámicas de corto, mediano y largo plazo. Según Carrión, la importancia de los centros históricos radica en la posibilidad de preservar y potenciar la memoria - para generar sentidos de identidad por función y pertenencia- $\mathrm{y}$ de convertirse en plataforma de innovación del conjunto de la ciudad (Carrión 2009).

Para el caso del Centro Histórico de Quito (CHQ), se aprecian ciertas problemáticas que ponen en peligro su adecuado funcionamiento y trascendencia en el futuro. Se continúa dando prioridad al tráfico automotor, la inseguridad se incrementa y sus dinámicas de uso continúan 
siendo regidas por la economía terciaria y la informalidad. Es por ello que surgen varias interrogantes respecto a las áreas y espacios públicos del Centro Histórico, ¿son seguros y placenteros los parques y plazas?, ¿son diversos, equitativos, e inclusivo?, ¿existe una articulación donde se priorice al peatón?

Es por ello que el presente estudio centra su objetivo en mostrar problemáticas existentes a nivel del CHQ en general, y en el área de estudio en específico, que evidencian la desarticulación de espacios públicos de alto valor simbólico, patrimonial e identitario; así como la amenaza de que esta importante zona se convierta en un lugar meramente turístico y escenográfico.

Hacer un llamado de atención a los habitantes y autoridades de la ciudad desde la visión y trabajo de la academia para contribuir de forma positiva a la consolidación de estas importantes áreas de la ciudad, la articulación de sus espacios públicos mediante la implementación de acciones sensibles que contribuirán de manera sostenible a revitalizar y poner en valor de uso espacios de gran significado y con ello lograr que las personas ya sean residentes o visitantes se apropien, utilicen y protejan estos lugares.

\section{METODOLOGÍA Y MATERIALES}

El trabajo consistió en una investigación urbana, dirigida a la zona más céntricas del $\mathrm{CH}$ de la ciudad de Quito, específicamente en el área fundacional y enlace de cinco importantes plazas de la ciudad, realizada con estudiantes de cuarto nivel de la carrera de Arquitectura en la Universidad Tecnológica Equinoccial, mediante la observación y levantamiento de datos directamente sobre el área urbana identificada y su población residente. Fue realizada en tres etapas; la primera referida al entendimiento y familiarización del área seleccionada. Un segundo momento para realizar un diagnóstico con énfasis en las variables sociodemográficas, accesibilidad, movilidad, espacios públicos y el paisaje urbano histórico. Por último, una fase encaminada a plantear algunos aspectos que fueron considerados como prioritarios para el mejoramiento funcional de un área de alto valor.

Para ello fueron consultadas $\mathrm{y}$ verificadas, diferentes fuentes y documentos que abordan la problemática del CHQ, así como diferentes planes de intervención implementados. Se revisaron además las fuentes cartográficas e informaciones levantadas por las instituciones competentes, 
que sirven de base para el análisis de las diferentes variables. La investigación fue respaldada por la indagatoria de campo, levantamientos fotográficos y planimétricos, complementada por las entrevistas $y$ consultas a los habitantes y visitantes de la zona.

Con el fin de lograr una mejor comprensión y evaluación de la problemática, la información recopilada fue procesada, mediante métodos cualitativos y cuantitativos que corroboran y apoyan los resultados obtenidos.

\section{DESARROLLO}

\section{El concepto de Centro Histórico}

Desde finales del siglo $\mathrm{XX}$, se han realizado planteamientos sobre esta importante área de la ciudad, enfocados hacia la importancia de mirarla, no solo por sus valores culturales o patrimoniales, sino como el conjunto de valores sociales, económicos, urbanos, ambientales, culturales y todos entrelazados son lo que la hacen distinguible y a su vez parte del todo que es la ciudad.

El Coloquio de Quito, celebrado en 1977, dejó en claro en el documento Carta de Quito, que la conservación de los centros históricos no se relaciona solamente con el patrimonio edificado, sino con el mejoramiento de la calidad de vida de sus habitantes "los centros históricos no solo son patrimonio cultural de la humanidad, sino que pertenecen en forma particular a todos aquellos sectores sociales que lo habitan".

Según Carrión (2007), las transformaciones en estas áreas de la ciudad se manifiestan social y urbanísticamente 1o que puede ser apreciado en fenómenos como la fragmentación, segregación y degradación de estos espacios y por consiguiente en la pérdida de ingresos $y$ afectaciones económicas de los territorios y sus habitantes.

Se destruyen valores y se consume suelos de forma desmedida. Se produjeron dos situaciones generalizadas: por un lado, el entorno de los centros históricos terminó por absorber recursos sin convertirse en un factor dinamizador de nuevas acciones (a excepción del turismo); por otro, generó el efecto de la expulsión de la población residente y de menores recursos (Carrión 2007). El Centro Histórico debe convertirse en "la plataforma de innovación del conjunto de la urbe y en objeto de deseo de la ciudad posible", (Carrión 2005, p. 38).

A la hora de caracterizar al centro, Castells plantea que es un núcleo lúdico, en el sentido de que hay concentración de lugares de entretenimiento, diversificación y 
ocio, pero también de vivienda (Castells, 2004).

\section{Caracterización del CHQ}

El Centro Histórico de Quito fue fundado por Sebastián de Benalcázar en el año 1534 y se convirtió en capital del país en el año 1830.

Se encuentra ubicado en el centro sur de la ciudad, en la provincia de Pichincha, Ecuador, cuenta con 14 barrios y es el mayor conjunto patrimonial de América Latina, contiene alrededor de 130 edificaciones monumentales y más de 5.000 inmuebles registrados como patrimonio histórico (Empresa Pública Metropolitana de Gestión de Destino Turístico, 2011); fue declarado Patrimonio Cultural de la Humanidad por la UNESCO en 1978. "El nombramiento se basó en el ensamble del centro histórico, considerado sui géneris y armónico para la época, en donde las acciones del hombre y la naturaleza se juntaron para crear una obra única y trascendental" (García, 2013). (Citado por Oviedo 2014).

Es un área urbana que alcanza las 376 hectáreas, con una trama vial que se adaptó a la topografía existente sus características históricas, culturales y geográficas lo sitúan como un referente para la identidad del pueblo ecuatoriano, y un patrimonio de carácter estratégico para el desarrollo económico del país (ver figura 1).

En el lugar interactúan residentes y visitantes se demuestra una gran dinámica social en la que conviven distintas clases sociales, por lo que es posible afirmar la existencia de conflictos causados por las desigualdades sociales (Ilustre Municipio de Quito 1992).

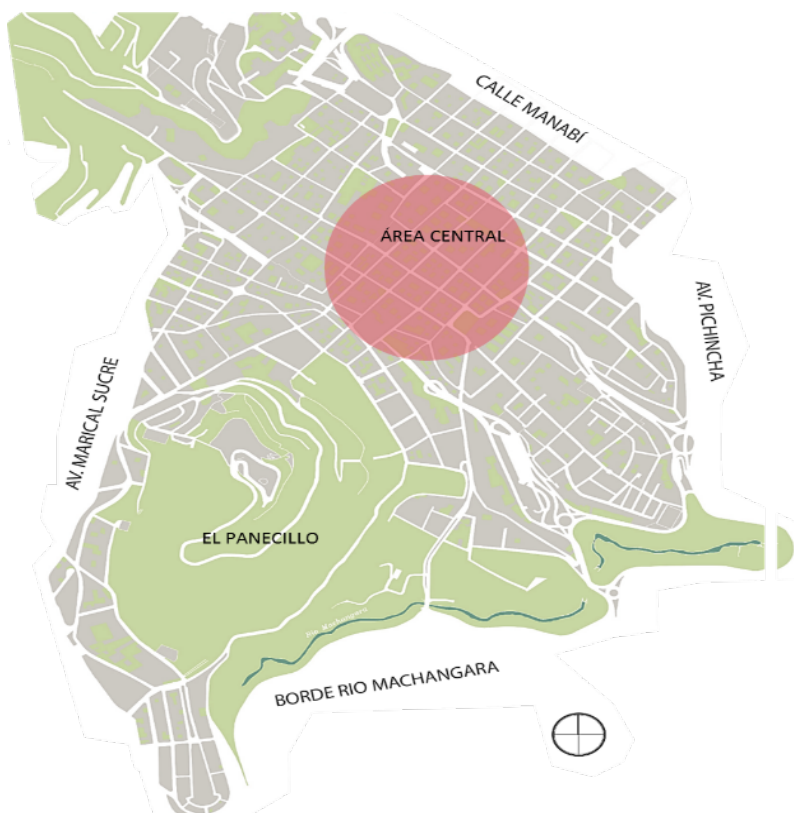

Figura 1. Mapa del Centro Histórico con énfasis en su área central y de mayor afluencia.

Fuente: Estudiantes 4to nivel, asignatura Introducción a la ciudad, Universidad Tecnológica Equinoccial.

Este trabajo centra su atención en el área más central del $\mathrm{CHQ}$, conformada por un circuito de relaciones que enmarca las Iglesias San Francisco, La Merced, San Agustín, Santo Domingo, y La Catedral. Cada una de ella se encuentra ubicada en plazas que han tomado el nombre de la 
institución religiosa que las preside, lo que le otorga carácter simbólico en estrecha relación con la cultura e identidad quiteña. Las conexiones, movilidad y accesibilidad a ellas poseen características distintivas que hacen de este lugar un atractivo indiscutible (ver figura 2).

Plaza de San Francisco lugar de gran importancia histórica en ella se asentaba un espacio de encuentro e intercambio de productos conocido como tiangues en la época hispánica se destacan el convento, iglesia y capilla de San Francisco, también la casa Gangotena ubicada en un extremo de la plaza convertida en hotel boutique, y a la instalación del restaurant Tianguez.

Plaza de la Merced regida por el convento e iglesia de La Merced, se convirtió en el proyecto ícono de la recuperación del $\mathrm{CH}$, importante sitio en la actualidad que concentra una fuerte actividad comercial.
Figura 2. Área Central del Centro Histórico de Quito con alta afluencia de personas.

Fuente: Estudiantes 4to. nivel asignatura introducción a la ciudad Universidad Tecnológica Equinoccial.

\section{Plaza de la Independencia o Plaza} Grande constituye uno de los espacios públicos más importantes de la ciudad las edificaciones que la rodean como el Palacio Arzobispal, el Palacio Hidalgo (actual hotel Plaza Grande), el edificio Pérez Pallares (Municipio del Distrito Metropolitano de Quito, el palacio de Carondelet sede del gobierno ecuatoriano).

Plazoleta de San Agustín se encuentra a un lado del atrio de la iglesia del mismo nombre $y$ un pequeño espacio que es intensamente utilizado por la población,

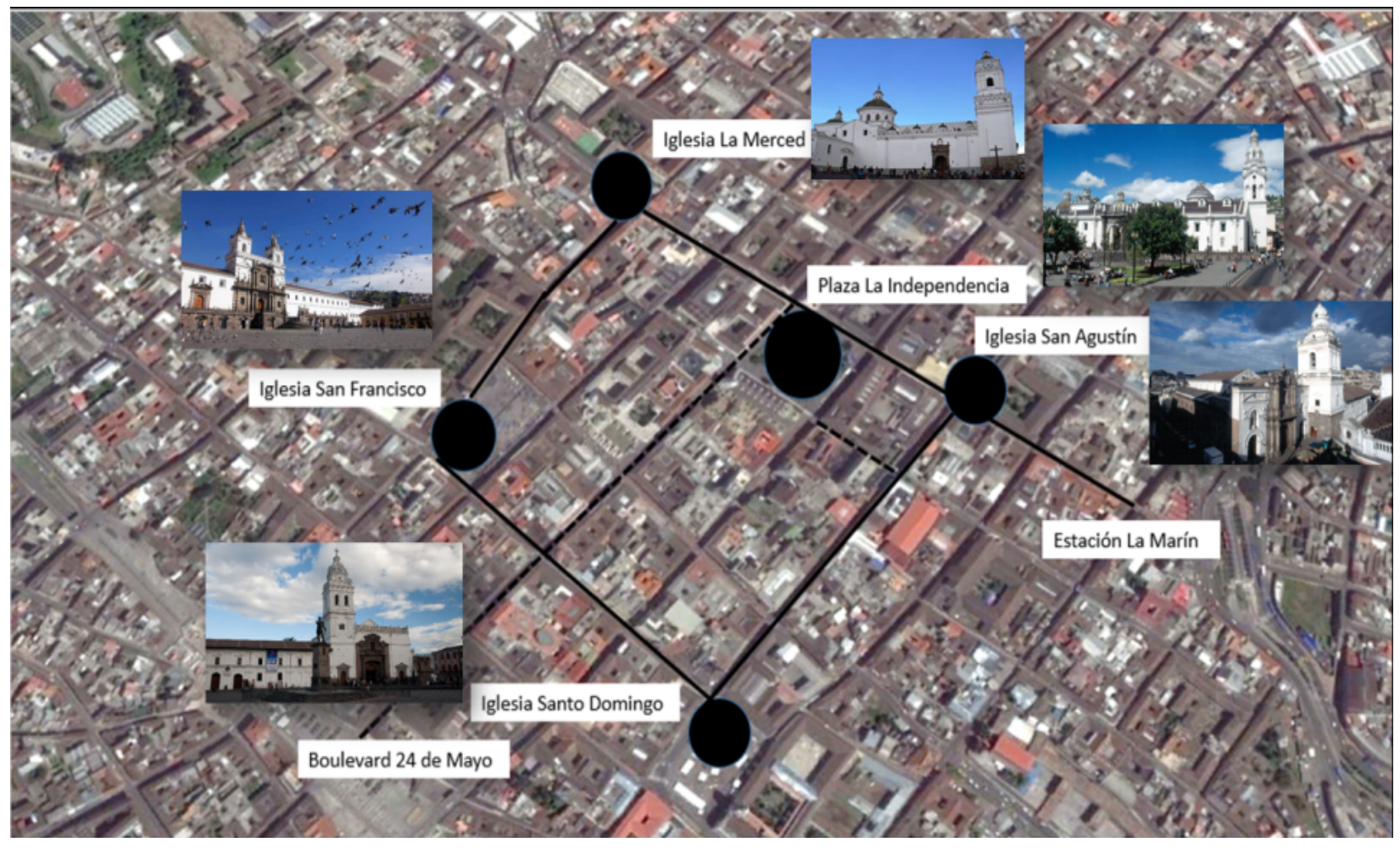


como permanencia y descanso o simple paso de un lugar a otro. Se asocia al importante y concurrido eje peatonal de la calle Chile.

Plaza de Santo Domingo compuesta por el convento e iglesia de Santo Domingo se distingue por su importancia comercial en la época colonial, se convirtió en el lugar de entrada desde el sur de la ciudad y sitio de encuentro desde el siglo XIX, y en la actualidad sitio de confluencia por la parada del Trole Bus.

CHQ y su área central poseen un alto significado no solo para Ecuador, sino para toda la región de América Latina. El mismo ha sido objeto de múltiples intervenciones, todas ellas encaminadas a lograr su revitalización y permanencia como hito $\mathrm{y}$ testigo tangible de la historia e identidad ecuatoriana.

\section{Intervenciones en el CHQ}

En la década de los noventa se estructuró el Plan Maestro de Rehabilitación Integral de las Áreas Históricas de Quito, que constituye un punto de referencia sobre la problemática del CHQ, a partir de cuatro campos de estudio: histórico, socioeconómico, urbano y arquitectónico (Cifuentes 2008).

Plan Maestro de Rehabilitación Integral para las Zonas Históricas de Quito (1991): $\mathrm{Su}$ objetivo principal fue proponer una política general de rehabilitación de zonas históricas de la ciudad, generar soluciones puntuales a problemas tales como conflictos sociales, deterioro del patrimonio arquitectónico y ambiental, a través del mejoramiento de las condiciones de vida de los habitantes, reduciendo las distancias económicas y sociales que se expresan a nivel de territorio.

Plan de Rehabilitación del Centro Histórico-BID Ira fase (1994): Se planteó como objetivo conservar y rehabilitar el patrimonio del $\mathrm{CH}$, devolviéndole su importancia funcional, revitalizando las actividades comerciales y de servicios tradicionales, rehabilitar sus edificios históricos, mejorar aceras, señalizaciones, haciéndolo más atractivo para los visitantes interesados en su conjunto histórico y cultural.

Plan Especial del Centro Histórico (2003): Conjuntamente con la cooperación técnica de la Junta de Andalucía plantean que el proceso de mejoramiento del CHQ debe formar parte de un plan general para la ciudad, ya que esta zona de la ciudad se constituye en el eje de la nacionalidad ecuatoriana. Se consideró que el principal problema de este núcleo es el abandono de las funciones residenciales y por ello sus propuestas estuvieron encaminadas a 
estimular el mejoramiento de las condiciones de la vivienda y de los servicios conexo alcanzando para el año 2010, aproximadamente $\quad 16.000$ habitantes (Municipio del Distrito Metropolitano de Quito 2003, p. 64).

Establece una orientación hacia la heterogeneidad de usos de suelo que combine de forma equilibrada la residencia, el comercio, la producción, las oficinas de servicios públicos y privados, así como los espacios para la cultura, el ocio, la recreación y el turismo. (Municipio Distrito Metropolitano de Quito 2003, p. 72).

Plan $Q$ (2003): Se reconoce a Quito como una ciudad que ofrece historia $\mathrm{y}$ patrimonio por lo que se decide crear un plan para la gestión del turismo que establece un proyecto de definición de usos de suelo que distinga entre áreas patrimoniales, culturales, comerciales, gastronómicas, hoteleras, residenciales, espectáculos y la llegada a futuro de centros universitarios además de un plan de regulación del transporte, peatonalización y estacionamientos.

Plan de Rehabilitación del Centro Histórico-BID 2da fase (2004): En este plan los componentes desarrollados fueron sostenibilidad social, desarrollo urbano y espacio público, involucrar a la sociedad en el cuidado del espacio público.

Plan Equinoccio 21 (2004-2025): Se planteó la revitalización integral del CHQ, dotación de infraestructura, servicios públicos, transporte y conectividad moderna y eficiente, la recuperación integral del uso residencial, así como la puesta en valor de los elementos patrimoniales. Dentro de este plan se elaboró el Plan Quito hacia el Bicentenario: Plan de Gobierno (2005-2009); en el que se previó implementar infraestructura básica y servicios directamente relacionados con el uso turístico, mejorar la calidad de vida de los residentes y visitantes del Distrito, recuperación de barrios emblemáticos como La Ronda, Cumandá, el Tejar, San Roque, y la construcción, rehabilitación y remodelación de infraestructura turística y hotelera, y la regeneración urbana de sectores deteriorados, entre ellos San Blas, Panecillo.

Plan $Q$ 2012: Declaración del Centro Histórico de Quito como Zona Especial Turística (2012). Plan de Gestión de la Zona Especial Turística del Centro Histórico de Quito 2013. Este plan abre las posibilidades de reenfocar la gestión de esta zona emblemática de Quito desde el sector turismo como una prioridad de uso y de dinamización económica, para impulsar el ordenamiento del territorio y de las actividades relacionadas, en base a la definición de la estructura del espacio turístico. 
Durante más de 20 años esta importante zona de la ciudad ha sido objeto de múltiples intervenciones, las cuales han abarcado variados objetivos como: conservar $\mathrm{y}$ rehabilitar el patrimonio del $\mathrm{CH}$, mejoramiento de las condiciones de vida de los habitantes, revitalizar las actividades comerciales y de servicios tradicionales, heterogeneidad de los usos de suelo y el fomento de la función residencial. Más recientemente se le da un rol protagónico al desarrollo y gestión turística del área como principal promotor de su desarrollo a futuro.

Sin embargo, no se ha podido alcanzar una correcta articulación de los espacios públicos como estructurador de las áreas urbanas y actores claves de la interacción social, asimilación y aceptación por parte de los habitantes.

Otros aspectos medulares como el tema del despoblamiento y las dificultades en la movilidad tanto peatonal como vehicular no han podido ser solucionados, lo que atenta contra la eficiencia y competitividad del lugar.

Este trabajo se ha enfocado sobre una parte del CHQ asociado a importantes plazas y parques, donde la afluencia de personas es grande, y además son espacios de alto reconocimiento internacional y nacional, por lo que analizar su funcionamiento, dinámica e identificar sus falencias son metas prioritarias a alcanzar.

Se manifiestan una multiplicidad de problemas que atentan contra el correcto funcionamiento a nivel urbano de esta zona y a muy corto plazo influirán en su dinámica y eficiencia de uso como área atractiva de la ciudad y del país. Se destacan por su nivel de incidencia los relacionados con las variables demográficas, accesibilidad y articulación de los espacios públicos en pos de priorizar el uso y apropiación por parte de los habitantes $\mathrm{y}$ visitantes.

La visión clásica de los centros históricos sujetos a políticas de conservación del patrimonio cultural, como ha sido el caso de Quito, ha conspirado para la revitalización urbana de la zona y ha producido la expulsión de sus habitantes, con consecuencias de marginalización y pérdida de los valores de convivencia ciudadana, transformándose en la mayoría de los casos en escenografías para el turismo. (Municipio del Distrito Metropolitano de Quito 2013, p. 22)

El planeamiento y la gestión territorial relacionada con el Área Patrimonial correspondiente al CHQ estarán dirigidos a promover la generación de oferta de vivienda en áreas patrimoniales, consolidar el espacio público seguro, conectado y de calidad, mejorar la conectividad y accesibilidad de las áreas patrimoniales con el entorno urbano y equilibrar la dotación de equipamientos $\mathrm{y}$ servicios. (Municipio del Distrito Metropolitano de Quito 2011, p. 42).

A pesar de estos planteamientos, en el tiempo que ha transcurrido en 2017 
continúan evidenciándose problemas que deben ser atendidos integralmente.

\section{Problemas identificados:}

\section{Disminución poblacional}

\section{del Centro Histórico de Quito}

La disminución de personas en el lugar ha sido una realidad que comenzó a evidenciarse desde los años noventa, una pérdida de habitantes que se han desplazado a otros lugares por diferentes causas. Según datos extraídos de los censos de 2001 y 2010 , la población disminuye, se identifica un porcentaje del $2,6 \%$ de decrecimiento en dicho período. Inés del Pino (2013) manifiesta que en 1990 la población del Centro Histórico de Quito fue de 81.384 habitantes, y 20 años después se redujo a 40.913, casi a la mitad.

Gráfico 1. Decrecimiento progresivo poblacional del CHQ.

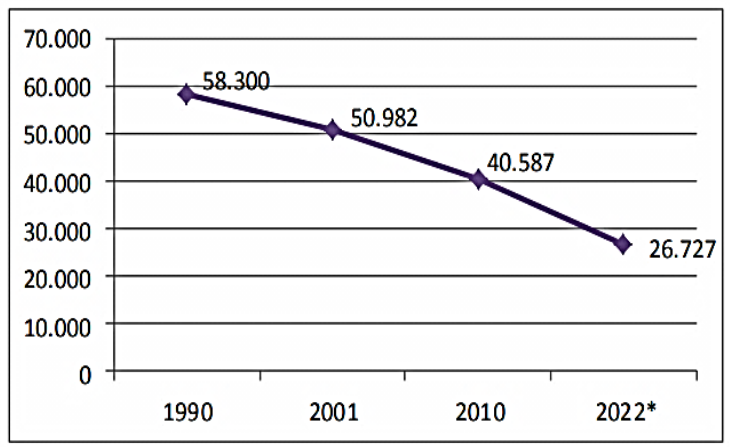

Fuente: Subsecretaría de Hábitat y asentamientos Humanos, (MIDUVI) 2011

Las proyecciones de las tendencias de crecimiento al 2022, prevén que se mantendrá la dinámica para esta área, en donde el número de pobladores seguirá en proceso de reducción (Municipio del Distrito Metropolitano de Quito 2011). El CH se desocupa y la pérdida de población residente es cada vez mayor.

Según el Municipio del Distrito Metropolitano de Quito en su diagnóstico estratégico de las áreas históricas, la población residente en el CHQ presenta un alto porcentaje de edad adulta (28\%, comprendida en la edad entre 36 a 64 años y un $8 \%$ que sobrepasa los 65 años). Estas características asociadas a la composición por edades poseen implicaciones a escala de la ciudad ya que se generan nuevas necesidades y mayores especificidades de diseño para los espacios, así como nuevos usos. La población residente presenta un alto porcentaje de desempleo, acompañada de falta de identidad con el sector.

Este es considerado un problema grave y preocupante, pues amenaza la existencia de esta área urbana, sin población que viva en el lugar, poco a poco, quedará en abandono convirtiéndose en un lugar muy peligroso y del cual el deterioro y la destrucción de sus valores será indetenible. Son los habitantes y residente los que le aportan vida, singularidad y permanencia del lugar en el tiempo. 
A pesar de que estos análisis se refieren a todo el $\mathrm{CH}$, su influencia sobre el área de estudio es notable, ya que de continuar disminuyendo los habitantes residentes, esta área tendrá menos personas viviendo en sus edificaciones y fuera de los horarios en los que funciona la actividad comercial el lugar estará vacío.

\section{Dinámicas de uso}

El sitio es muy concurrido con gran demanda de uso debido a que es identificada como una centralidad de tipo administrativa, comercial y de servicios. Presenta una alta concentración de actividades terciarias, donde los servicios comerciales definen con marcada preferencia los horarios laborables y se afectan las dinámicas del lugar sobre todo en horas de la noche, ya que prevalecen los usos inscriptos entre las 8 de la mañana y las 7 de la noche, lo que provoca que en horarios nocturnos prevalecen pocas actividades, locales cerrados, baja circulación de peatones y se genera la inseguridad y la posibilidad para la delincuencia.

Es la actividad comercial la que juega el rol protagónico como uso y apropiación del espacio e impacta de forma más notoria el lugar, se aprecia alta ocupación de las plantas bajas de las edificaciones, vendedores ambulantes de todo tipo ubicados en vías, parques y plazas.

Gráfico 2. Comportamiento de los sectores económicos en el CHQ.

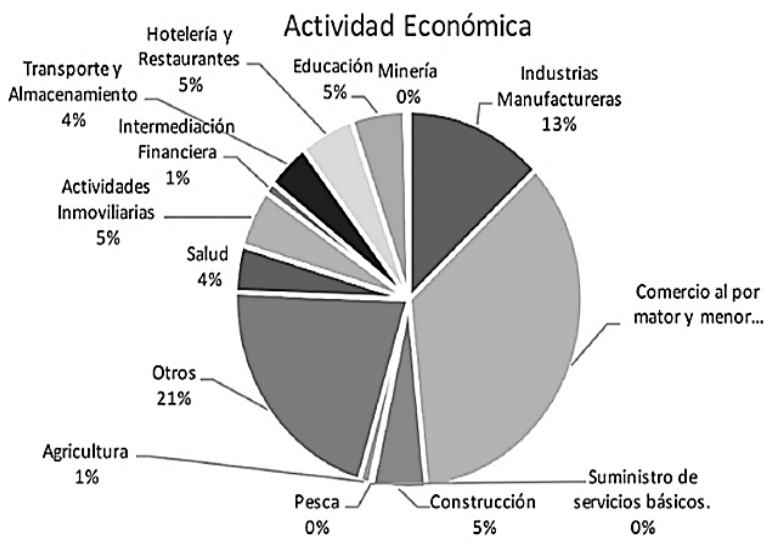

Fuente: Estudiantes 4to. nivel asignatura introducción a la ciudad Universidad Tecnológica Equinoccial. INEC.

En cuanto a la afluencia resulta importante señalar la cantidad de personas que transitan por múltiples razones el área. La actividad turística genera gran concurrencia de población flotante, considera unos 500.000 turistas internacionales por año y cerca de un millón de nacionales, además de las personas que utilizan la zona como tránsito hacia otras áreas de la ciudad.

\section{Deficiente accesibilidad y movilidad}

Existen diversas formas de llegar a la zona de estudio, peatonalmente, en transporte público, buses, el Trole, taxis y el transporte privado. En la mayoría de los casos turismo juega un rol predominante, como la manera 
en que se puede disfrutar y utilizar esta área, pero también es ruta para moverse de un lugar a otro de la ciudad. La accesibilidad y la movilidad son un problema bien marcado, la propia configuración física y geográfica del lugar, sus calles estrechas, sus aceras pequeñas y a veces ausentes, su relieve pronunciado y caracterizado por fuertes pendientes son condicionantes muy fuertes que condicionan su desenvolvimiento.

A todo esto, se une la falta de organización de la movilidad, un creciente congestionamiento vehicular por calles que no fueron diseñadas para semejante número de autos, una competencia de tipos de transporte público y privado que en todos los casos entorpecen al peatón y la peatonalización en detrimento del uso y apropiación del espacio público (calles, parques y plazas), como contenedor prioritario de las relaciones sociales.

El excesivo tráfico automotor genera además otros problemas; entre ellos: el ruido, la contaminación por gases, mala calidad del aire e inseguridad para las personas.

Las calles Cuenca, García Moreno, Venezuela y Guayaquil como principales ejes de la zona de estudio, son claros ejemplos de congestionamiento donde vehículos y peatones se disputan por la prioridad de utilizar el espacio, y se generan grandes conflictos que no solo afectan el correcto funcionamiento, sino que crean una imagen de caos (ver figura 3 ).

Fig. 3. Congestionamiento y conflictos entre peatones y vehículos en las calles del CHQ.
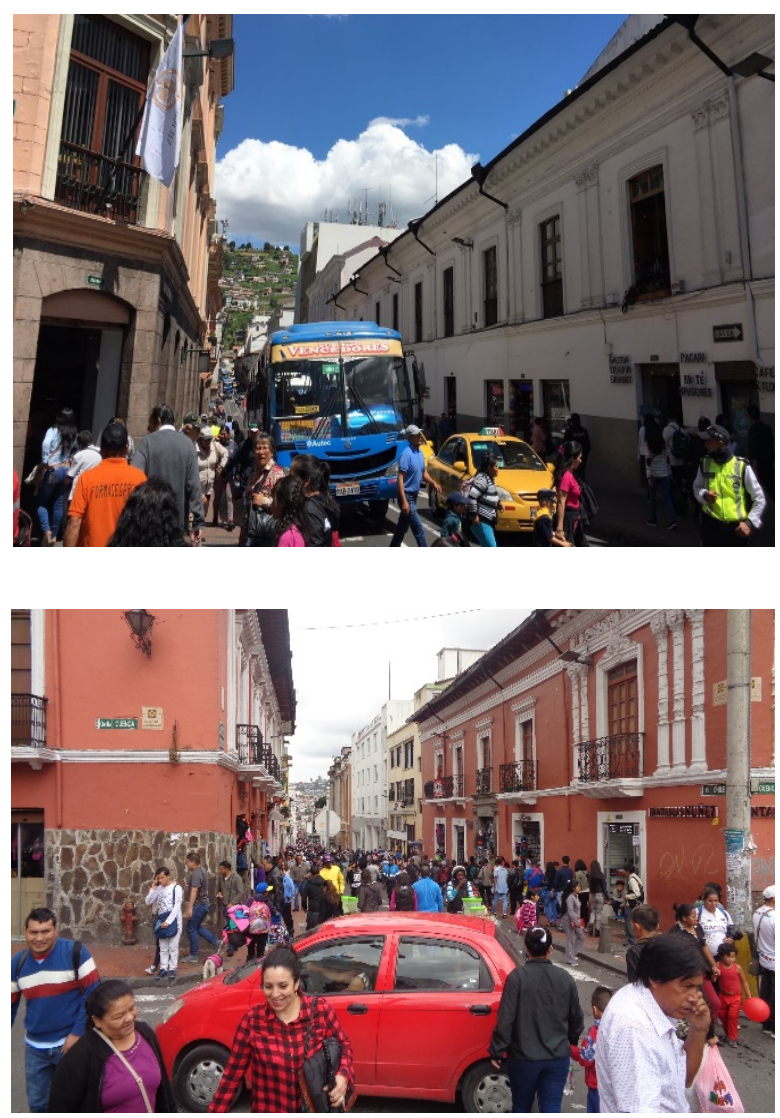

Fuente: Estudiantes 4to nivel asignatura introducción a la ciudad Universidad Tecnológica Equinoccial.

La zona de estudio está atravesada por las calles Venezuela y García Moreno, ubicada en ambos lados de la Plaza de La Independencia y por la cual circula un intenso tráfico automotor, la calle Guayaquil es el eje que recorre el Trole, con paradas importantes en la Plaza de Santo Domingo, casi un $50 \%$ de los viajes motorizados utilizan el sector como ruta diaria, lo que 
resulta en un problema de alto impacto ya que no está diseñado para asumir el creciente tráfico y las presiones generadas, tanto por el tipo y número de vehículos como de personas que circulan por el lugar. Es conocido que por el área cruzan diariamente varias líneas de buses del servicio público, según datos de la Agencia Metropolitana de Tránsito (ATM). Se producen grandes conflictos para entrar y salir, y a todo esto se suman los insuficientes espacios para estacionarse, las disponibilidades en este aspecto no dan abasto a la demanda.

\section{Falta de articulación de los espacios públicos más relevantes}

Se destaca un circuito de alto valor que enlaza importantes hitos arquitectónicos, identificado por cinco importantes plazas del $\mathrm{CH}$, cada una con una historia, papel simbólico y arquitectura que las hace distintas y atrayentes por sí mismas.

- Iglesia y Plaza de San Francisco.

- Iglesia y Plaza de La Merced.

- La Catedral y Plaza de la Independencia.

- Iglesia y plazoleta de San Agustín.

- Iglesia y Plaza de Santo Domingo.

Las calles que conforman el perímetro de enlace de estos importantes espacios públicos son las calles: Cuenca, Chile, Guayaquil, Simón Bolívar, y las calles García Moreno y
Venezuela que bordean la Plaza de La Independencia.

Estos importantes espacios públicos, poseen carácter identitario, protagonizado por una obra arquitectónica predominantemente religiosa, sin embargo, resulta complejo disfrutar de un recorrido establecido, dinámico, seguro, complementado por usos de suelo atractivos, mobiliario urbano eficiente, iluminación $\mathrm{y}$ áreas verdes agradables.

Los principales problemas identificados en relación directa con el espacio público en el circuito seleccionado para el estudio son:

- Deficiente peatonalización con recorridos interrumpidos que no dan continuidad ni enlazan los espacios públicos relevantes de este contexto.

- Baja intensidad de uso con dinámicas que priorizan los horarios del día y dejan abandonados los de la noche.

- Presencia en el espacio público de indigencia y marginalidad, lo que aumenta la sensación de inseguridad entre la población residente y los visitantes.

- Déficit en arbolado, áreas verdes y jardinería.

- Insuficiente mobiliario urbano que apoye las necesidades de las personas que recorren el lugar. 
- Uso inadecuado de los espacios públicos, en algunos casos es excesiva y en otros se subutiliza.

- Insuficiente limpieza del espacio público, recolección de basuras, falta de mantenimiento permanente de la imagen y paisaje del centro histórico.

Insuficiente aprovechamiento de los valores paisajísticos del contexto

En 2011, se adoptó la Recomendación de UNESCO sobre Paisaje Urbano Histórico, en la misma se estableció que este tipo de paisaje comprende la topografía, la geomorfología y las características naturales del sitio, su entorno edificado, tanto histórico como contemporáneo, sus infraestructuras de superficie y subterráneas, sus espacios verdes y jardines, sus planos de ocupación de suelo y su organización del espacio, sus relaciones visuales y todos los elementos constitutivos de la estructura urbana. Incluye las prácticas y valores sociales, culturales, económicos y las dimensiones inmateriales del patrimonio, diversidad e identidad. (Declaración de la UNESCO, 2011), lo cual permite determinar que el paisaje es la imagen de la ciudad,

En este caso en específico y como resultado de la revisión de los planes implementados en el CHQ, se puede apreciar que ha existido mucha preocupación por el patrimonio edificado y su preservación, así como dar prioridad a la actividad turística, pero el paisaje no ha sido suficientemente abordado. Se refiere con exactitud a que los aspectos recomendados como características naturales, espacios verdes y jardines, organización del espacio y sus relaciones visuales, no han sido suficientemente abordados, se puede apreciar la invasión constructiva del Panecillo, el desorden y conflictividad entre vehículo y peatón, no apropiación de visuales y espacios existentes que servirían para ubicar áreas de estar con mobiliario urbano adecuado.

Sin embargo, está demostrado que recuperar los valores del paisaje urbano histórico deviene en calidad para el hábitat y la vida de las personas.

Para el área de estudio analizada es importante resaltar las visuales que se generan desde los espacios públicos (parques y plazas) que lo conforman, en cada uno existe un telón de fondo de carácter natural o más antropizado o asociado a la arquitectura, que pueden y deben ser mejor aprovechados, se distinguen con mucha fuerza las visuales al Panecillo.

\section{RESULTADOS Y DISCUSIÓN}


"El CHQ ha sido y en parte continúa siendo, a pesar de políticas equivocadas en ese sentido, el espacio privilegiado de la cultura y la religiosidad popular" (Kingman y Goetschel, 2005).

Ante los problemas anteriormente identificados y descritos es necesario garantizar la mixtura de los usos de suelo asociados a esta importante área urbana, y este debe ser eficiente, diverso, equitativo e inclusivo, como una manera de ampliar las dinámicas, horarios y tiempo de uso del espacio.

Se requiere trabajar con la gente que vive y dinamiza el área estudiada, conocer sus aspiraciones y deseos respecto al lugar donde viven, ya que para muchos turistas constituye el mayor atractivo, su gente y sus tradiciones, no obstante, en la generalidad de las intervenciones planteadas y ejecutadas la participación no ha sido todo lo integral $\mathrm{y}$ efectiva que se requiere en un entorno de tan alto valor simbólico, identitario $\mathrm{y}$ patrimonial.

Es posible trabajar en lineamientos relacionados con los impuestos y las facilidades de pago para las acciones de mantenimiento y conservación de las viviendas patrimoniales, ya que en muchos casos los dueños son núcleos de bajos ingresos incapacitados de asumir los costos que estas edificaciones demandan.

El funcionamiento de una red interconectada y articulada de espacios públicos, donde predominen los intereses del peatón, las relaciones sociales, el disfrute del paisaje, se constituye en la actualidad en una disfunción del área estudiada, ya que en la generalidad de los espacios es muy fuerte los conflictos generados por la movilidad peatonal y vehicular.

La inserción de elementos de mobiliario, vegetación e iluminación han de contribuir a la creación de un sentido de apropiación y disfrute de estos lugares llenos de identidad, significado y simbolismo y generar una cultura y sensibilidad por espacios únicos de altos valores ambientales, arquitectónicos y urbanos, a pesar de ello, no en todos los espacios están presentes, por ejemplo es evidente la ausencia de un diseño que identifique al lugar, no son suficientes los recipientes o contenedores para la basura, la señalización y la señalética es insuficiente y de bajo valor en su diseño.

Generar nuevos paisajes como resultado de jerarquizar visuales y conducir los flujos hacia puntos de interés es algo totalmente insuficiente, ejes visuales de un altísimo valor paisajístico son poco utilizados. El eje de la García Moreno que conecta con el 
boulevard de la 24 de Mayo y mira al Panecillo es hoy uno de los ejes más congestionados de la zona.

La accesibilidad y movilidad por priorizar el servicio al peatón y disminuir el tráfico vehicular en el circuito estudiado debe ser una prioridad.

La calle Cuenca en el tramo que conecta a la Plaza de La Merced y la Plaza San Francisco; la calle García Moreno y la calle Venezuela deben ser peatonalizadas, potenciando las plantas bajas de los edificios con usos culturales y recreativos que favorezcan la conexión entre plazas y parques, aumenten los horarios de uso y disfrute y se genere una zona donde el peatón sea el verdadero protagonista.

Figura 4. Plaza de la Merced con alta aceptación por habitantes y visitantes y una visual paisajística de fondo.
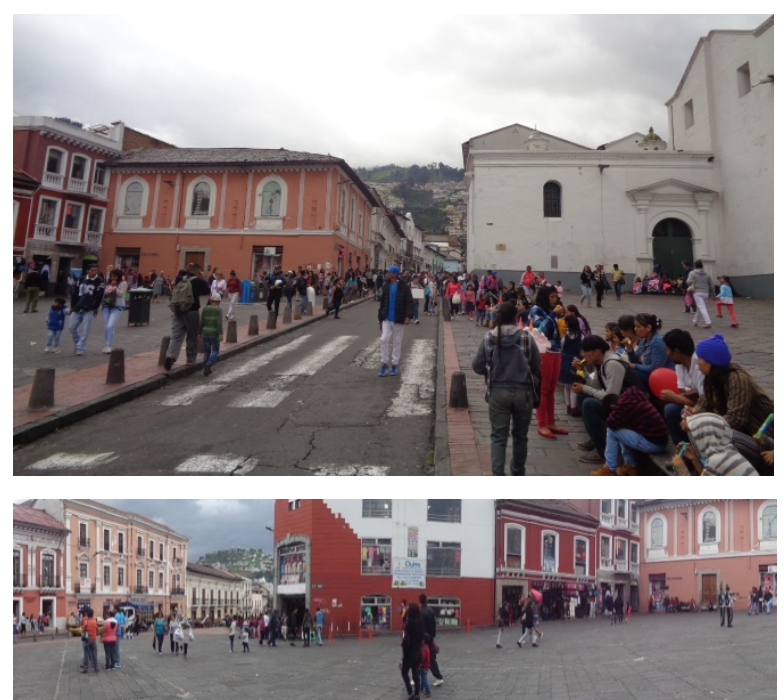

Fuente: Estudiantes 4to nivel asignatura introducción a la ciudad Universidad Tecnológica Equinoccial.

En términos generales el sector analizado se constituye en el área más céntrica del $\mathrm{CHQ}$, de ahí que mejorar su funcionamiento y actuar sobre los ejes, espacios públicos, paisaje urbano histórico, accesibilidad y movilidad podría contribuir a que las personas permanezcan en el lugar y su estancia sea más placentera y segura.

\section{CONCLUSIONES Y RECOMENDACIONES}

Los centros históricos, su paisaje y la riqueza patrimonial que lo caracterizan se constituyen hoy en día en un valioso recurso, un derecho de las personas que lo habitan y lo visitan, en el cual hemos de apoyarnos para alcanzar la meta de la ciudad sostenible.

Poner en valor estos espacios, mediante planes que contribuyan a difundir y proteger la cultura e identidad del lugar deben incluir de manera prioritaria los aspectos que incluyen el paisaje y los atributos que le son inherentes como el relieve, los hitos naturales como El Panecillo en conjunto con su arquitectura, tradiciones y costumbres.

La puesta en valor de estos espacios debe asimilar e interpretar la diversidad morfotipológica y funcional, conjuntamente difundir y proteger la cultura e identidad del 
lugar, para ello el trabajo participativo con la población, las instituciones competentes debe ser un aspecto insoslayable.

La apropiación y preservación del patrimonio edificado y su paisaje como valor existente, distintivo y atrayente de los ambientes urbanos debe contribuir a generar una cultura y sensibilidad por espacios únicos de altos valores ambientales, arquitectónicos y urbanos.

\section{REFERENCIAS}

\section{BIBLIOGRÁFICAS}

Carrión, F. (2007). Financiamiento de los centros históricos de América Latina y El Caribe. Quito: FLACSO-E.

Carrión, F. (2009). "El centro histórico como objeto de deseo". En F. Carrión y L. Hanley (eds.). Regeneración y revitalización urbana en las Américas: Hacia un estado estable (pp. 35-57). Quito: FLACSO- E / USAID.

Carta de Quito. (1977). Coloquio sobre la preservación de los centros históricos ante el crecimiento de las ciudades contemporáneas. Quito: UNESCO / PNUD.

Castells, M. (2004). La cuestión urbana. México DF: Siglo XXI.
Cifuentes, C. (2008). La planificación de las áreas patrimoniales del Centro Histórico de Quito. Quito: FLACSO-E.

Colectivo de autores. (2008). "Revitalización de Centros Históricos La arquitectura de hoy, entre la ciudad histórica y la actual". Ciudad de México 27, 28 y 29 de octubre de 2008 .

Municipio del Distrito Metropolitano de Quito. Diagnóstico Estratégico-Áreas Históricas. Problemática en $\mathrm{CHQ}$ y áreas patrimoniales

Oviedo, M. S. (2014). “Centro histórico de Quito: Cambios en la configuración residencial y usos de suelo urbano asociados al turismo. Tesis de maestría en Asentamientos Humanos y Medio Instituto de Estudios Urbanos y Territoriales, Pontificia Universidad Católica de Chile.

Pino, I. D. (2010). Centro histórico de Quito, una centralidad urbana hacia el turismo. Quito: Abya-Yala.

Quito, M. D. (2003). Plan Especial del Centro Histórico. Quito.

Quito, M. D. (2004). Plan Equinoccio 21. Quito.

UNESCO. (2011). "Recomendación sobre el paisaje urbano histórico, con inclusión de un glosario de definiciones". Instrumentos normativos. 
Page 18 of 18 